\title{
The inhibitory role of si-UBB delivered by degradable dendrimers-based lipid nanoparticles in ovarian cancer
}

\author{
Shanrong Shu ${ }^{1 \dagger}$, Xiaoping $\mathrm{Liu}^{2 \dagger}$, Ming Xu${ }^{3}$, Yaduan $\mathrm{Lin}^{4}$ and Ruiman $\mathrm{Li}^{1 *}$
}

\author{
${ }^{*}$ Correspondence: \\ butterfly1705@126.com \\ †Shanrong Shu and Xiaoping \\ Liu contributed equally to \\ this work \\ ${ }^{1}$ Department of Gynecology \\ and Obstetrics, The \\ First Affiliated Hospital \\ of Jinan University, 613 \\ Huangpu Road West \\ 510630 Guangzhou, People's \\ Republic of China \\ Full list of author information \\ is available at the end of the \\ article
}

\begin{abstract}
Objective: RNA interference holds tremendous potential in the treatment of malignant tumors. However, efficient and biocompatible delivery methods are needed for systemic delivery of small interfering RNA (siRNA). In this study, we explored the delivery efficiency and therapy effect of si-UBB-5A2SC8 in ovarian cancer.

Methods and materials: Si-UBB-5A2SC8 nanoparticles were successfully prepared according to the established procedure and the characteristic of nanoparticles was determined by digital laser scanning. Flow cytometry and confocal analysis demonstrated si-UBB was efficiently transfected to cell by the delivery of 5A2SC8 complexes. The in vitro gene knockdown efficiency of ubiquitin B was demonstrated by RT-qPCR and Western blot analysis, which was further verified by the inhibition of proliferation and migration of ovarian cancer cells. Accumulative efficiency of si-UBB-5A2SC8 nanoparticles was investigated in BALB/c mice bearing SKOV3-GFP tumor xenograft. In vivo imaging was adopted to test the accurate location of the nanoparticle in the tumor. The feature of the tumor and pivotal organ was determined. TUNEL and caspase-3 expression was used to analyze the underling mechanism of the inhibition effect.

Results: The average size and the zeta potential of the si-UBB-5A2SC 8 was (150 \pm 11$)$ $\mathrm{nm}$ and $-(20 \pm 4) \mathrm{mV}$, respectively. Transmission electronic microscopy showed the nanoparticle was near-spherical with the mean size of (100 \pm 15$) \mathrm{nm}$. Flow cytometry and confocal microscopic images demonstrated 5A2SC8 complex could successfully deliver Cy5.5-siRNA to the cytoplasm of ovarian cancer cells. qRT-PCR and western blot demonstrated the mRNA and protein expression of ubiquitin $B$ was decreased to $62.5 \%$ and $36.5 \%$ of the control group, which was accompanied with the decreased proliferation and migration ability in si-UBB-5A2SC8-transfected cells. Ex vivo fluorescence imaging demonstrated $5 \mathrm{~A} 2 \mathrm{SC} 8$ complex could successfully carry siRNA to the tumor lesion and exert the inhibition effect, which was verified by the decreased tumor weight and increased apoptosis and caspase-3 expression in mice treated with Si-UBB-5A2SC8.
\end{abstract}

Conclusions: 5A2SC8 complex was a safe and efficient gene delivery vehicle and ubiquitin B was a potential target for the ovarian cancer targeted therapy.

Keywords: siRNA, Nanoparticles, Ovarian cancer, Ubiquitin-B, 5A2SC8 


\section{Introduction}

Epithelial ovarian cancer remains the most lethally gynecological malignancy due to its advanced stadge at diagnosis and the quick development of chemotherapy resistance, which is the sixth leading cause of cancer-related death in women among entire world (Bray et al., 2018). Though many gynecological oncologists had improved surgical techniques and made great advancement in chemotherapy, survival rate hardly increased in past decades. The major obstacle is that invisible metastases has occurred in the other part of the body, which cause incurable tumor relapse and underspin an imperative need for new therapeutic strategies (Alvero et al., 2017).

Gene target therapy has been developed over the past decades resulting from the comprehensive understanding of molecular and genetic alteration in cancer. RNA interferences (RNAi) mediated by short interfering RNA (siRNA) has enormous therapeutic potential for the treatment of cancer owing to its effective downregulation of designated gene (Kim and Rossi, 2007). Genes regulating of proliferation or angiogenesis are found to be mutated in cancer, which results to uncontrolled proliferation and these genes are a promising target for the gene silencing therapy. UbiquitinB encoded by UBB gene is a member of ubiquitin/proteasome system, which accounts for extensive protein degradation and is considered as a regulator of many physiological processes, such as signal transduction, DNA repair, chromosome maintenance, transcriptional activation, cell cycle progression and cell survival (Oh et al., 2013). UBB is reported to be overexpressed in many solid tumor including ovarian cancer (Oh et al., 2013). Therefore, we inferred that UBB suppression via siRNA technique would provide effective anti-tumor role on human ovarian cancer. In fact, siRNA knockdown of the UBB gene has been reported to enhances the radio-sensitivity of human cervical squamous cell carcinoma by regulating DNA damage-binding protein 2 (Zhou et al., 2020).

However, RNAi therapeutics are still hindered by some issues for further application in clinic, such as rapid degradation in systemic circulation and poor cellular uptake efficiency (Ozcan et al., 2015). Approaches to enhance the delivery of siRNA focus on finding a suitable delivery platform, which enables biocompatibility, a high loading capacity, protection of siRNA during transport and a high targeting ability. Thus, lipid or polymer-based nanocarrier has been used to wrapped RNAi to conquer these problems (Barata et al., 2016), especially for dendrimers-based lipid nanoparticles with chemical groups to bind small RNAs and nanoparticle (NP)-stabilizing hydrophobicity, which combine to encapsulate RNA molecules inside stable NPs and release small RNAs into the cytosol after endocytosis. Therefore, dendrimers-based lipid nanoparticles can reduce the non-specific distribution in normal tissue and target tumors through enhanced permeability and retention effect.

In the present study, we used modular degradable dendrimers as the gene delivery system to transfect ovarian cancer cell SKOV3 with siUBB nanoparticle. The feature of the complexes was characterized and anti-tumor potent of the complexes against human ovarian cancer was assessed both in vitro and in vivo. Our results suggested that the established siUBB nanoparticle has a potential application in ovarian cancer gene therapy. 


\section{Materials and methods \\ Reagent}

$5 \mathrm{~A} 2 \mathrm{SC} 8$ which was the core component of the nanoparticle was synthesized and provide by Dr. Daniel Siegwart (the comprehensive cancer center, the Southwestern Medical Center of Texas University). 2-Dioleoyl-sn-glycero-3-phosphoethanolamine (DOPE), cholesterol and DMG-PEG2000 were purchased from Sigma-Aldrich. Caspase-3(Asp175) antibody was purchased from Cell Signal. Cy5.5-labeled siRNA (Cy5.5-siRNA) was supplied by Suzhou Ribo Life Science Co., Ltd. Ubiquitin-B siRNA (siUBB) and control siRNA were purchased from Sigma-Aldrich. The sequences for Control siRNA, si-UBB and Cy5.5-siRNA are following: Control siRNA sense: 5 '-TTC TCC GAA CGT GTC ACGT-3', anti-sense: 3'-ACG TGA CAC GTT CGG AGAA-5'. si-UBB sense: 5'-GUA UGC AGA UCU UCGUGAA-3', anti-sense: 3'-CAU ACG UCU AGA AGC ACUU-5'. Cy5.5-siRNA sense:5'-UUCUCCG AACG UGUCACGU-3', antisense:3'- ACG UGA CAC GUU CGG AGAA -5'.

\section{Cell lines and animal}

Human ovarian carcinoma SKOV3 cells stably transfected with GFP was kindly provided Dr. Daniel Siegwart and cultured in DMEM supplemented with $0.1 \%$ gentamycin (Sigma-Aldrich) and 10\% FBS (Gibco) in a humidified atmosphere containing 5\% $\mathrm{CO}_{2}$ at $37{ }^{\circ} \mathrm{C}$. We bought 20 female BALB/c nude mice (average weight $20 \mathrm{~g}$ ) with the age of 5-6 weeks from Shanghai SLAC Laboratory Animal Co., Ltd, and all the mice were kept under specific pathogen-free conditions. The mice experienced treatment and were monitored health and behavior twice a week, after 8 times of treatment, the mice were killed by cervical dislocation. The animal experiments were assessed and approved by the Ethics Committee of Jinan University with the registration number of IACUC-20181231-02.

\section{Cell viability}

SKOV3-GFP were plated in 96-well opaque plates with the density of 5000 cells per well. After $24 \mathrm{~h}$, the cells were $80 \%$ confluence and transfected with various concentration of siRNA-Ctrl-5A2SC8 or si-UBB-5A2SC8 for $72 \mathrm{~h}$. The number of viable cells was calculated by quantifying ATP using CellTiter-Glo cell viability luminescent assay kit (Promega, Madison, WI). $100 \mu \mathrm{L}$ of CellTiter-Glo reagent was prepared according to the instruction of the manufacture and added to each plate. The plates were incubated for $10 \mathrm{~min}$ in the dark, then we measured the luminescence using a FLUO star OPTIMA microplate reader.

\section{Migration analysis}

We seeded the cells in 6 -well plates with the concentration of $2.5 \times 10^{5} /$ well. In the next day, when the cells reached approximately 90 percent of confluence, we transfected the cells with si-UBB-5A2SC8 or siRNA-Ctrl-5A2SC8 at the siRNA concentration of $0.2 \mathrm{ng} / \mathrm{ul}$. After transfection, the cells were artificially scratched with a pipette tip of $0.2 \mathrm{~cm}$ in width, and cultured in medium containing 1\% FBS. At $0 \mathrm{~h}, 48 \mathrm{~h}$ and 
$72 \mathrm{~h}$, images of the scratch wounds were captured. Cell migration was measured as the new scratched width relative to the original scratched width.

\section{Real-time quantitative RT-PCR}

Total RNA was extracted using RNA simple Total RNA Kit (TIANGEN, Haidian, Beijing, China), and was then reverse-transcribed by using a Prime Script ${ }^{\mathrm{TM}}$ RT reagent Kit (TaKaRa, Dalian, Liaoning, China). The amount of cDNA was normalized to GAPDH (Sangon Biotech (Shanghai) Co., Ltd). The primes of the genes were shown as the following ubiquitin B, forward: 5'-CTT TGT TGG GTG AGC TTG TTT GT-3', and reverse: 3'-GAC CTG TTA GCG GAT ACC AGG AT-5'. GAPDH forward, 5'AGA AGG CTG GGG CTC ATTTG-3', and reverse: 3'- AGG GGC CAT CCA CAG TCTTC-5. All-in-One ${ }^{\mathrm{TM}}$ qPCR mix (GeneCopoeia, Inc., Rockville, MD, USA) was used to detect the expression level of UBB. The real-time RT-PCR conditions were following: $95{ }^{\circ} \mathrm{C}$ for $10 \mathrm{~min}$ followed by 40 cycles of $15 \mathrm{~s}$ at $95{ }^{\circ} \mathrm{C}, 15 \mathrm{~s}$ at $60{ }^{\circ} \mathrm{C}$ and $30 \mathrm{~s}$ at $72{ }^{\circ} \mathrm{C}$. Relative quantification of each gene was calculated by using the $2^{-\Delta \Delta C T}$ method and normalized to the control value to obtain percent fold changes.

\section{Western blot analysis}

The total protein was obtained using radioimmunoprecipitation assay lysis and extraction buffer (Pierce; Thermo Fisher Scientific, Inc.) and the concentration was determined by BCA protein assay kit (Pierce; ThermoFisher Scientific, Inc.). Equal amounts of the protein samples were separated by $10 \sim 12 \%$ SDS-PAGE. Membranes were incubated with primary antibodies against ubiquitin B (Cell Signaling Technology, P4D1, USA). Horseradish peroxidase-labeled anti-mouse antibody, $\beta$-actin was used as a secondary antibody (Beijing Zhong Shan Biotech Co., Ltd. Beijing, China). Membranes were visualized using enhanced chemiluminescence (ECL; Millipore). The band of $\beta$-actin served as an internal control. Band density was quantified by Image J software.

\section{Preparation of nanoparticles (NPs) and NPs/siRNA complexes}

Nanoparticles were prepared as described previously (Cheng et al., 2018). 5A2SC8, DOPE, cholesterol, and DMG-PEG2000 were dissolved in 100\% ethanol with the concentration $50 \mathrm{ug} / \mathrm{ul}, 10 \mathrm{ug} / \mathrm{ul}, 5 \mathrm{ug} / \mathrm{ul}$ and $10 \mathrm{ug} / \mathrm{ul}$, respectively. Then the nanoparticle was formed by mixing 5A2SC8, DOPE, cholesterol, and DMG-PEG2000 with the volume ratio 1:0.429:1.596:0.269. The NPs was stored at $-20^{\circ} \mathrm{C}$ for use. For the formulation of NPs/siRNA complexes, according to the protocol determined by Dr. Daniel Siegwart, when the concentration of nanoparticle/siRNA was $1.65 \mathrm{ug} / \mathrm{ul}$, the nanoparticle had the best effect. First, we calculated the needed siRNA according to the protocol of our experiment, then according to the concentration of $1.65 \mathrm{ug} / \mathrm{ul}$, we got the amount of nanoparticles. Second, we diluted siRNA and nanoparticles with acid buffer $(10 \mathrm{nM}, \mathrm{pH} 4.0)$ when it was necessary, so we made the volume of siRNA was three times of nanoparticles. Last we mixed siRNA and nanoparticle by fast vortex and incubated for $15 \mathrm{~min}$ at room temperature. 


\section{Characterization of particle sizes and zeta potential}

The hydrodynamic diameter and zeta potential of siRNA-5A2SC8 nanoparticle were measured using a laser particle size analyzer (Zetasizer Nano ZS, Malvern, UK) at a wavelength of $633 \mathrm{~nm}$ with a constant angle of $173^{\circ}$. The morphology and particle size of siRNA-5A2SC8 NPs were determined by transmission electron microscopy images (H-6009IV; Hitachi Ltd., Tokyo, Japan).

\section{Cell internalization studies using confocal images and flow cytometry}

For confocal microscopic images, SKOV3-GFP were cultured overnight to reach $80 \%$ confluence. Then the cells were transfected with free fluorescent Cy5.5-siRNA or Cy5.5-siRNA loaded with 5A2SC8 $(1 \mathrm{mg} / \mathrm{ml})$ for $3 \mathrm{~h}$. We washed each well three times with PBS, and then fixed the cells with paraformaldehyde for $10 \mathrm{~min}$. 4,6-Diamidino-2-phenylindole (DAPI) was added to the cells for nuclear staining. Cellular internalization of the fluorescent Cy5.5-siRNA-loaded 5A2SC8 NPs was determined with LSM710 laser-scanning confocal microscope (Carl Zeiss), which was equipped with $405 \mathrm{~nm}$ and $514 \mathrm{~nm}$ laser for DAPI and Cy5.5, respectively. For flow cytometry analysis, we first treated each cell line with the fluorescent Cy5.5-siRNA-loaded 5A2SC8 NPs, then examined fluorescent density by Guava EasyCyte flow cytometer (Merck Millipore).

\section{Tissue distribution of nanoparticles}

To verify 5A2SC8 particle can deliver siRNA to tumor tissue, PBS, free Cy5.5-siRNA and Cy5.5-siRNA loaded with 5A2SC8 nanoparticles were injected into BALB/c mice with the dose of siRNA $1 \mathrm{mg} / \mathrm{kg}$. For the ex vivo imaging, the tumor tissue and major organs of mice were collected after $24 \mathrm{~h}$ of intraperitoneal injection. Excised organs were imaged by using a Bio-Real in vivo imaging system (Bio-Real Quick View 3000; Geneway International, Salzburg, Austria). Cy5.5 molecules were excited by a 675$\mathrm{nm}$ pulsed laser diode. The fluorescence emission at $710 \mathrm{~nm}$ collected was applied to assess the distribution of nanoparticles.

\section{In vivo orthotopic model of ovarian cancer and tissue processing}

Female BALB/c nude mice were kept in a specific pathogen-free housing facility. To produce tumors, SKOV3-GFP cells with $5 \times 10^{6}$ in 100 ul PBS were injected into the peritoneal cavity of the mice. First, we used 3 mice to test whether 5A2SC8 nanoparticle could deliver siRNA to tumor lesions. Next the tumor-bearing mice were randomly divided into three groups. Treatment began one week after the inoculation of cells. We treated the mice by ip injection of PBS, siRNA-Ctrl-5A2SC8, siUBB-5A2SC8 at the dose of siRNA $1 \mathrm{mg} / \mathrm{kg}$ every 3 days. After 8 times of treatment, the mice were killed. Tumors tissue or important organs were obtained and fixed in $4 \%$ paraformaldehyde or frozen in liquid nitrogen immediately. Weight of the tumor and mice was recorded.

\section{Histological analysis and TUNEL staining}

Slides (5um) were made from tumor tissue and pivotal organs. Hematoxylin and eosin $(\mathrm{H} \& \mathrm{E})$ staining was performed according to standard procedure. For 
immunohistochemistry analysis, slides were blocked with $3 \% \mathrm{H}_{2} \mathrm{O}_{2}$ for 15 min at room temperature, incubated with goat serum for $1 \mathrm{~h}$ at room temperature, and subsequently incubated with rabbit anti-mouse caspase- 3 antibodies at $4^{\circ} \mathrm{C}$ overnight. After washing with PBS for three times, the appropriate secondary antibody conjugated to horseradish peroxidase (HRP) was added. The slices were washed and counter stained with hematoxylin for $20 \mathrm{~s}$. All images were recorded in 5 random fields for each slide. Tissues were stained by terminal deoxynucleotidyl transferase-mediated deoxyuridine triphosphate nick-end labeling (TUNEL; green; Promega, Madison, WI). An apoptotic body was represented by green fluorescence. To calculate apoptotic cells, TUNEL-positive cells were determined in 10 random fields from five separate slides per group.

\section{Statistical analysis}

All assays were conducted three times and found to be reproducible. Data were assessed using software packages SPSS version 16.0. Data were expressed as the mean \pm SD. Comparisons among all groups were performed with the one-way analysis of variance test and the post hoc test with $\mathrm{S}-\mathrm{N}-\mathrm{K}$ (Student-Newman-Keuls) was used to determine the difference between two groups. $P<00.05$ was considered significant.

\section{Results}

\section{Preparation and characterization of siRNA-loaded nanoparticles}

The particle size and zeta potential of the nanoparticle were analyzed by dynamic light scattering (Zetasizer 3000, Malvern Instruments, Malvern, England). As shown in Fig. 1, the average size of the particle was $(150 \pm 11) \mathrm{nm}$, the zeta potential was - $(20 \pm 4)$ $\mathrm{mV}$. Transmission electronic microscopy by measurements of each nanoparticle and at

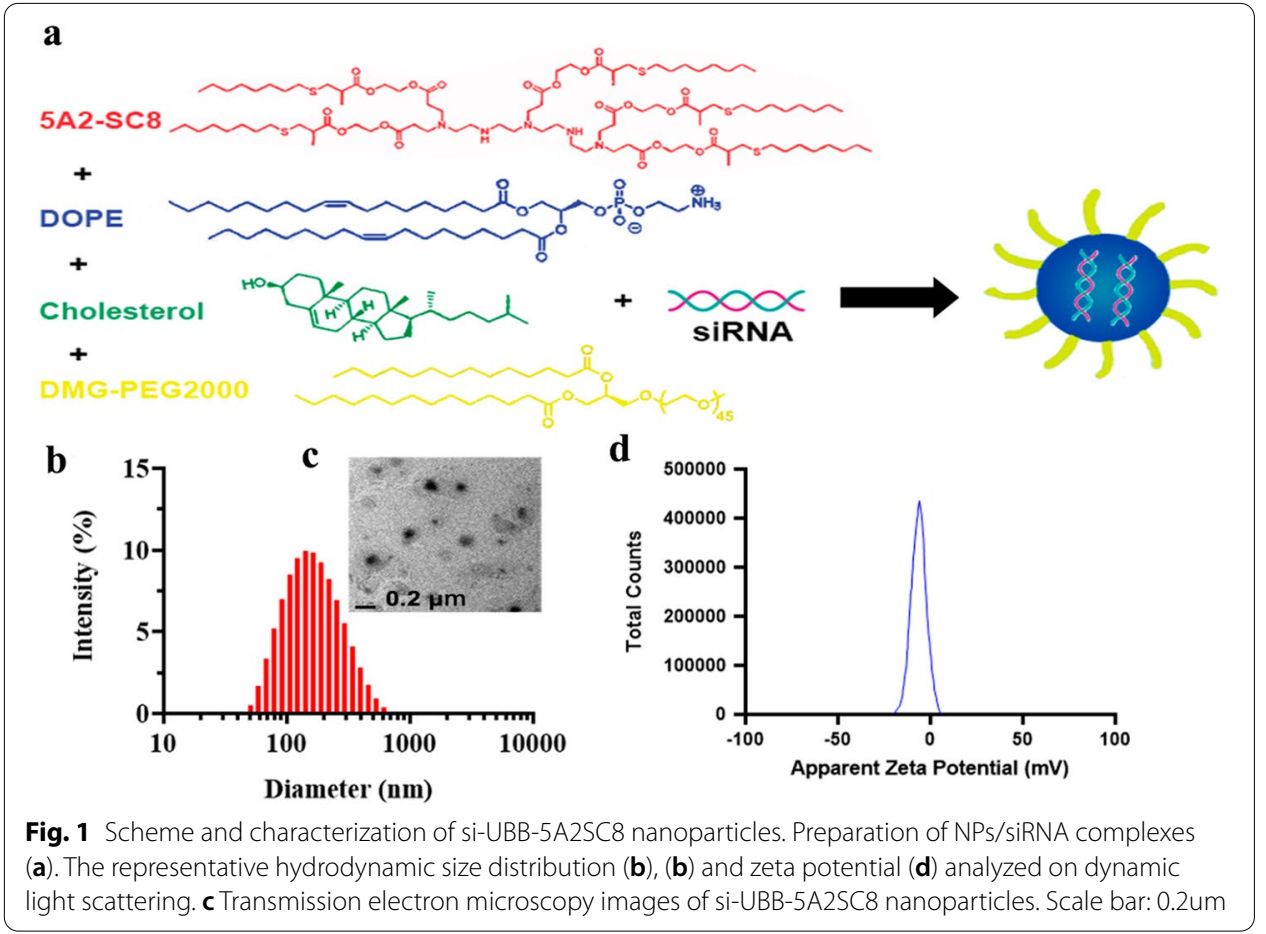


least 100 microcapsules per sample was determined, which has been adopted by many researchers (Pinho et al., 2010; Lees et al., 2013) was adopted to observe the morphology of the nanoparticle, it appeared near-spherical with the mean size of $(100 \pm 15) \mathrm{nm}$.

\section{Cellular uptake of Cy5.5-siRNA loaded with 5A2SC8}

To assess the cellular uptake of 5A2SC8 particle, the fluorescent Cy5.5-siRNA-loaded 5A2SC8 nanoparticles were treated to SKOV3-GFP cells. Flow cytometry analysis demonstrated that the increased fluorescent intensities in Cy5.5-siRNA-loaded 5A2SC8 treated cell lines indicated that 5A2SC8 NPs efficiently delivered the encapsulated Cy5.5-siRNA into ovarian cancer cell lines (Fig. 2a). The confocal microscopic images showed that Cy5.5-siRNA-5A2SC8 nanoparticles treated cells exhibited the intracellular fluorescence signals in the cytoplasm compartment, whereas there was little or no fluorescence in the nucleus (Fig. 2b). All these data demonstrate that siRNA-5A2SC8 nanoparticles can be substantially internalized into ovarian cancer cells, which guaranteed the downregulation effect of siRNA in cells.

\section{Suppression of si-UBB-loaded nanoparticles on ovarian cancer cells}

The downregulation efficiency of siUBB-5A2SC8 nanoparticles was investigated in ovarian cancer cells both in mRNA level and protein level. After $72 \mathrm{~h}$ of treatment with nanoparticle complexes, cell lysates were obtained. qRT-PCR and western blot was performed to determine the inhibition efficiency of siUBB-5A2SC8. As shown in Fig. 3a, compared with the PBS and si-Ctrl nanoparticle treatment group, the level of ubiquitin B mRNA was significantly downregulated, it was $62.5 \%$ less than the si-Ctrl nanoparticle treatment group. Meanwhile, the protein of ubiquitin B was significantly suppressed in si-UBB-5A2SC8 nanoparticle treatment group, which was decreased to $36.5 \%$ of the siCtrl nanoparticle treatment group (Fig. 3b, c). What's more, irrespective of the mRNA level and protein level, there was no significant difference between PBS and siRNA-Ctrl treatment group.
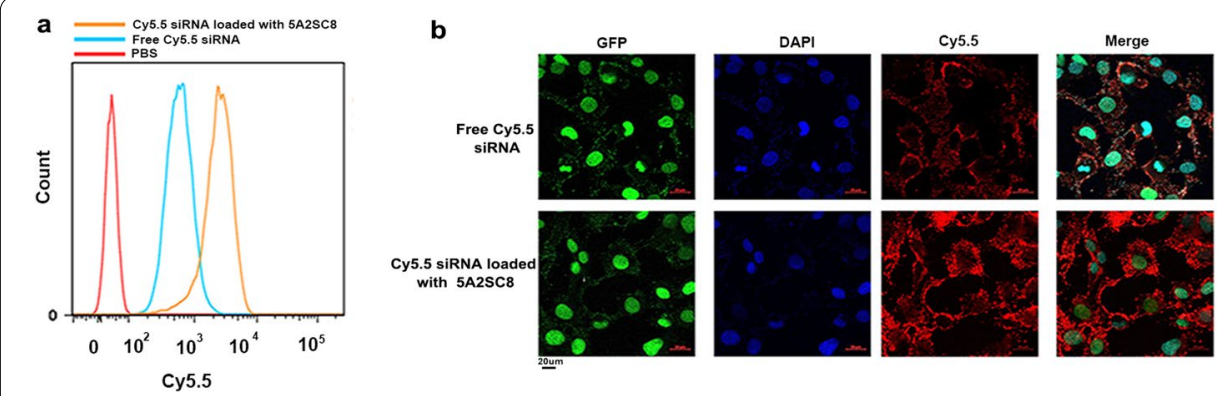

Fig. 2 Efficient cellular internalization of si-UBB-5A2SC8 nanoparticles in SKOV3-GFP ovarian cancer cells. a Flow cytometry was used to quantify the fluorescence intensity. The results showed the nanoparticle 5A2SC8 could efficiently deliver Cy5.5-siRNA to cytoplasm. Scale bar: 20um. b SKOV3-GFP cell were treated with free Cy5.5-siRNA or Cy5.5-siRNA loaded with 5A2SC8 for $3 \mathrm{~h}$, confocal microscope was used to analyze the fluorescence signal. GFP, Cy5.5-siRNA and DAPI dyes are represented by green, red and blue, respectively. Scale bar: $20 \mu \mathrm{m}$ 

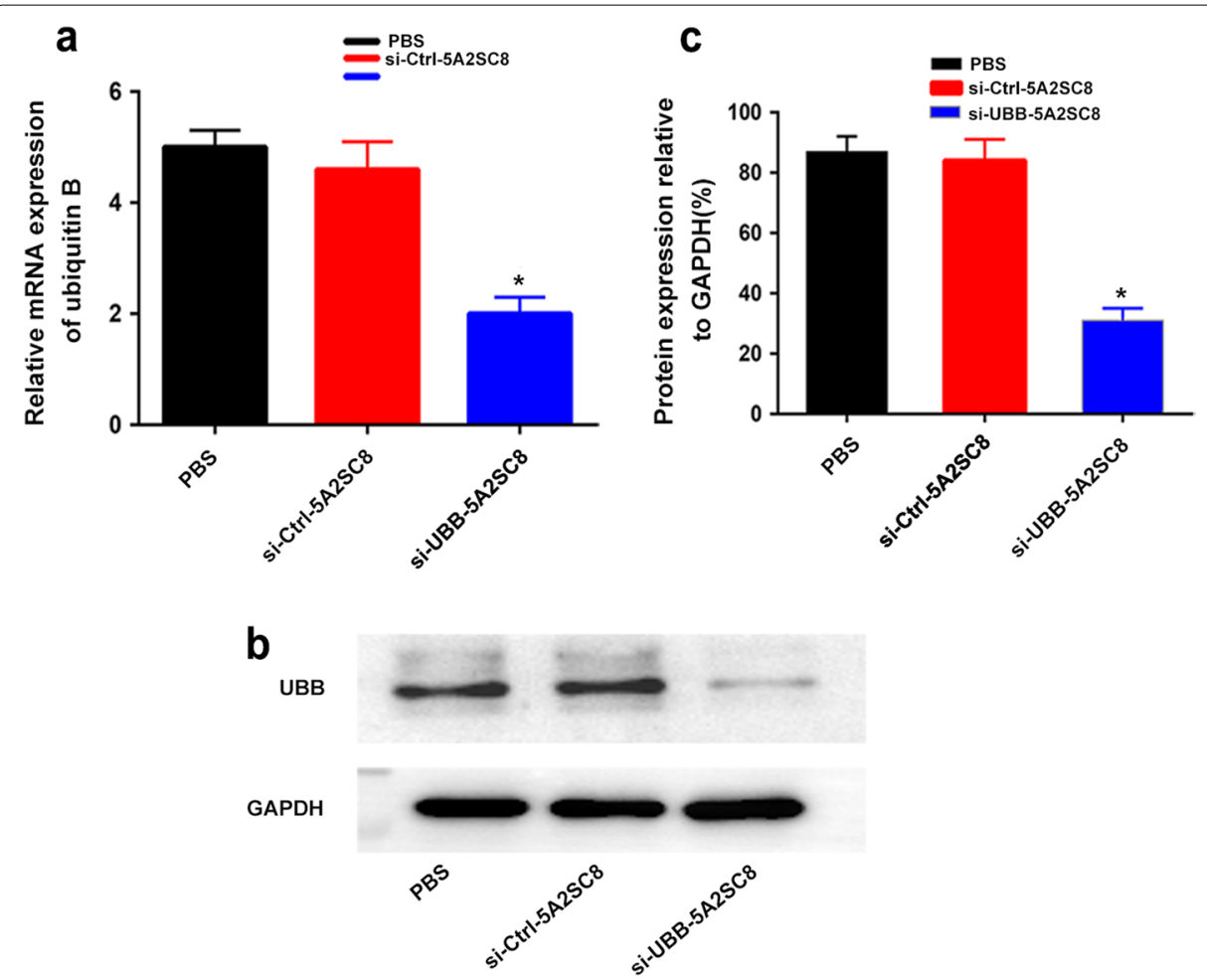

Fig. 3 Knockdown of ubiquitin B expression in SKOV3-GFP cells. a si-UBB loaded with 5A2SC8 could significantly inhibit mRNA expression, which decreased to $62.5 \%$ of the PBS and si-Ctrl nanoparticle treatment group. $\mathbf{b}, \mathbf{c}$ si-UBB loaded with $5 \mathrm{~A} 2 \mathrm{SC} 8$ significantly inhibited the protein expression of UBB, which decreased to $36.5 \%$ of the control. ${ }^{*} P<0.05$

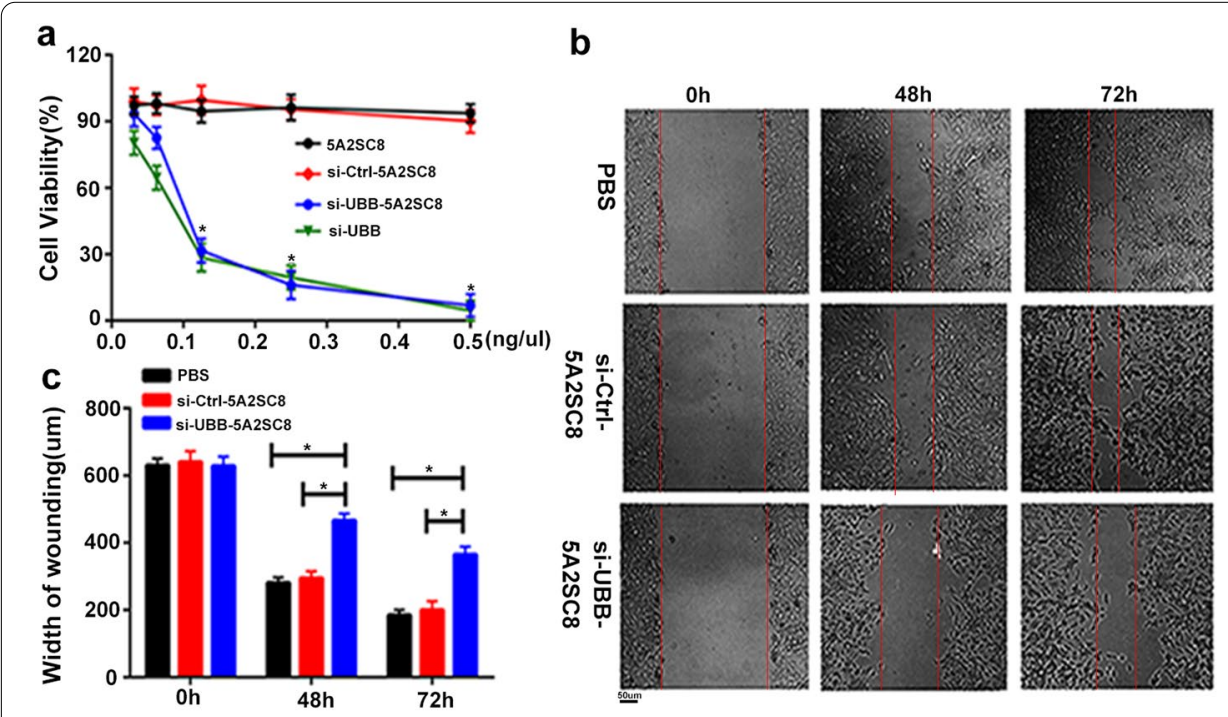

Fig. 4 Knockdown of ubiquitin B expression in SKOV3-GFP cells inhibited ovarian cancer cell proliferation and migration. a Downregulation of UBB significantly inhibited the proliferation of cells, meanwhile, nanoparticle 5 A2SC8 had little toxicity to cell. The viability of cells was above $90 \%$ when cells were treated with $5 \mathrm{~A} 2 \mathrm{SC} 8$ alone or $\mathrm{Ctrl}-5 \mathrm{~A} 2 \mathrm{SC} 8$. b, $\mathbf{c}$ The migration of cells was decreased when treated with si-UBB-5A2SC 8 for $48 \mathrm{~h}$ or $72 \mathrm{~h}$, the width of wound was significantly enlarged when compared with PBS and si-Ctrl-5A2SC8. ${ }^{*} P<0.05$. Scale bar: $50 \mu \mathrm{m}$ 


\section{si-UBB-loaded nanoparticles inhibit ovarian cancer cell proliferation and migration}

When we treated ovarian cancer cells only with 5A2SC8 particle and without any siRNA for $72 \mathrm{~h}$, we found the cell viability was above $90 \%$, which meant that the particle had minimal toxicity to cell (Fig. 4a). We treated ovarian cancer cells with si-UBB-5A2SC8 for $72 \mathrm{~h}$, the proliferation of SKOV3-GFP was significantly decreased compared with si-Ctrl-5A2SC8-treated group. Meanwhile, the inhibitory role was becoming more prominent with the increased concentration of si-UBB-5A2SC8. We also found the cell proliferation in si-UBB-5A2SC8 group and si-UBB group were comparable, which mean free si-UBB was not degraded in $72 \mathrm{~h} .48 \mathrm{~h}$ and $72 \mathrm{~h}$ after scratching, a wound-healing assay showed si-UBB-5A2SC8 transfected groups displayed a significantly reduced wound closure compared with si-Ctrl-5A2SC8 and PBS groups. Meanwhile, there was no significantly difference in the wound closure between si-Ctrl-5A2SC8 and PBS groups (Fig. 4b, c).

\section{Ex vivo fluorescence imaging}

Once nanoparticle wrapped with siRNA reached the tumor site, siRNA can exert antitumor effect. To testify the hypothesis, we adopted Cy5.5-siRNA-5A2SC8 to examine the distribution of nanoparticle complexes. Because the surface of the nanoparticles has fluorescent Cy5.5, when the nanoparticles were administrated into the tumor-bearing mice, it was easy to track their distribution in vivo. As seen in the ex vivo fluorescence images, GFP fluorescence was detected to confirm the tumor lesions (Fig. 5a). The red fluorescence demonstrated the distribution of the nanoparticles. The mice treated with PBS, there was no red fluorescent signal. In free Cy5.5-siRNA and Cy5.5-siRNA-5A2SC8 treated group, the red fluorescent signals were also detected in the liver, kidney and
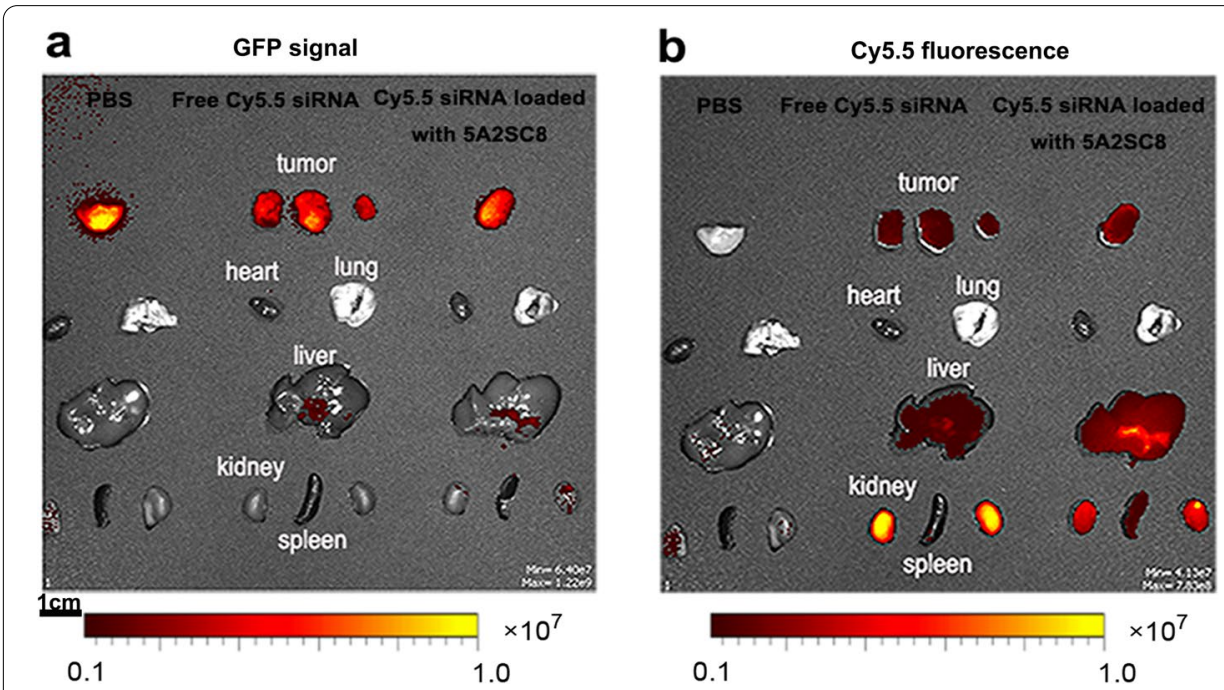

Fig. 5 Intraperitoneal ovarian tumor localization of Cy5.5 fluorescently labeled siRNA after administration of 5 A2SC8 carrier. BLAB/c mice were injected intraperitoneally with SKOV3-GFP. Two weeks after tumor cell injection, mice were peritoneally injected with phosphate buffered saline (PBS), free Cy 5.5-siRNA or Cy5.5-siRNA-5A2SC8 nanoparticle with the dose of siRNA $1 \mathrm{mg} / \mathrm{kg}$. Twenty-four hours later, mice were euthanized, and organs (heart, lungs, liver, spleen, kidneys, tumor) were removed. Tissues were imaged ex vivo for a GFP signal to identify luciferase expressing tumor cells and $\mathbf{b}$ Cy 5.5 fluorescence to visualize the biodistribution of siRNA-containing nanoparticles, respectively. Scale bar: $1 \mathrm{~cm}$ 
tumor. The fluorescent signal of the kidney was stronger in free Cy5.5-siRNA treated group compared with that in Cy5.5-siRNA-5A2SC8 treated group (Fig. 5b). All those results demonstrated that free Cy5.5-siRNA can be easily eliminated through kidney, and 5A2SC8 particle could protect Cy5.5-siRNA again degradation during in vivo circulation, which guaranteed the execution of our following experiment.

\section{Antitumor effects of si-UBB-loaded nanoparticles in vivo}

To verify the anti-tumor effects of nanoparticle complexes in vivo, a xenograft model of human ovarian cancer cells was constructed in female BALB/c nude mice. One week after inoculation with ovarian cancer cells, the volume of tumor was $100 \mathrm{~mm}^{3}$, then the mice received a peritoneal administration of si-UBB- $5 \mathrm{~A} 2 \mathrm{SC} 8$, si-Ctrl-5A2SC8 or PBS twice a week, and we measured the volume of mice every week(length"width"width/2). 8 times of administration, the tumor tissue and pivotal organ were taken out. As shown in Fig. 6, the average tumor volume was significantly decreased in si-UBB-5A2SC8 treated group (Fig. 6d). Tumor weight in si-UBB-5A2SC8, si-Ctrl-5A2SC8 or PBS treatment group was $0.42 \pm 0.09 \mathrm{~g}, 0.65 \pm 0.11 \mathrm{~g}$ and $0.66 \pm 0.12 \mathrm{~g}$, respectively (Fig. 6a, c). The weight of tumor in si-UBB-5A2SC8-treated group was significantly decreased compared with si-Ctrl-5A2SC8 and PBS group. No significant body weight changes were observed in the three groups (Fig. 6b). Meanwhile, there were no obvious histopathological abnormalities or lesions in the heart, live, lung, or kidney tissues observed for any of the treatment (Fig. 6e).

\section{Effect of si-UBB-5A2SC8 nanoparticle on ovarian tumor cell apoptosis}

To investigate the underlying mechanism for the reduced tumor growth in si-UBB5A2SC8-treated group, TUNEL assay and immunohistochemistry were performed to analyze whether si-UBB-5A2SC8 complex could induce apoptosis in tumor cells. As shown in Fig. 7, cells with bright green fluorescent staining (identified as apoptotic cells) could be observed in tumor tissue in si-UBB-5A2SC8-treated group, whereas the green fluorescent staining was rare in PBS or si-Ctrl-5A2SC8 treated group. Meanwhile, we detected the caspase- 3 expression, we found the expression of caspase- 3 was increased in si-UBB-5A2SC8-treated group compared with that in PBS or si-Ctrl-5A2SC8 treated group. All these results suggested that si-UBB-5A2SC8 complexes can prevent tumor progression by induction of apoptosis.

\section{Discussion}

RNAi-based therapy is an attractive method, but we must improve the delivery efficiency of siRNA in vivo (Aagaard and Rossi, 2007). For delivery efficiency of free siRNA without a vector is quite low, and most of the free siRNA is rapidly degraded when intravenous injection (Dragoni et al., 2016). In order to conquer these limitations, NP system with low toxicity and immunogenicity was widely adopted to deliver the siRNA (Castillo et al., 2018). As reported by Zhou et al. (2016), a lead dendrimer, 5A2SC8 could provide a broad therapeutic window and well tolerated in separate toxicity studies to provide a pronounced survival benefit in mice bearing MYC-driven tumors. In this work, we further validated its ability to deliver siRNA in ovarian cancer. Studies involving titration of phospholipid into cationic lipid formulations clearly showed that phospholipid was not 


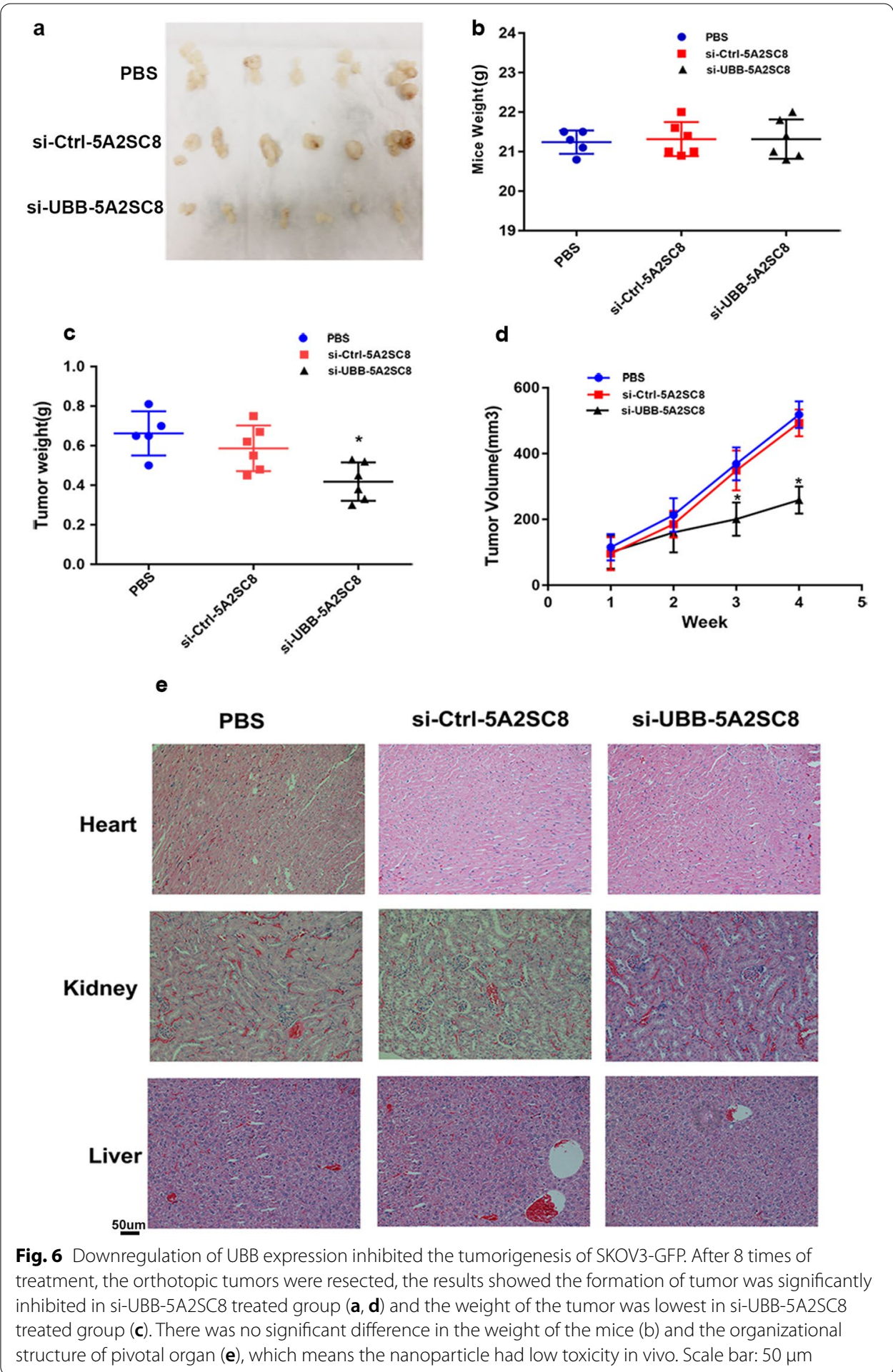

necessary for siRNA delivery but was required for mRNA deliver. Therefore, we used 5A2-SC8, DOPE, cholesterol and DMG-PEG2000 to construct nanoparticles.

In our study, 5A2SC8-based nanocarrier was successfully synthesized and was used to transfer siUBB to ovarian cancer cell SKOV3-GFP. The 5A2SC8 nanoparticle is 


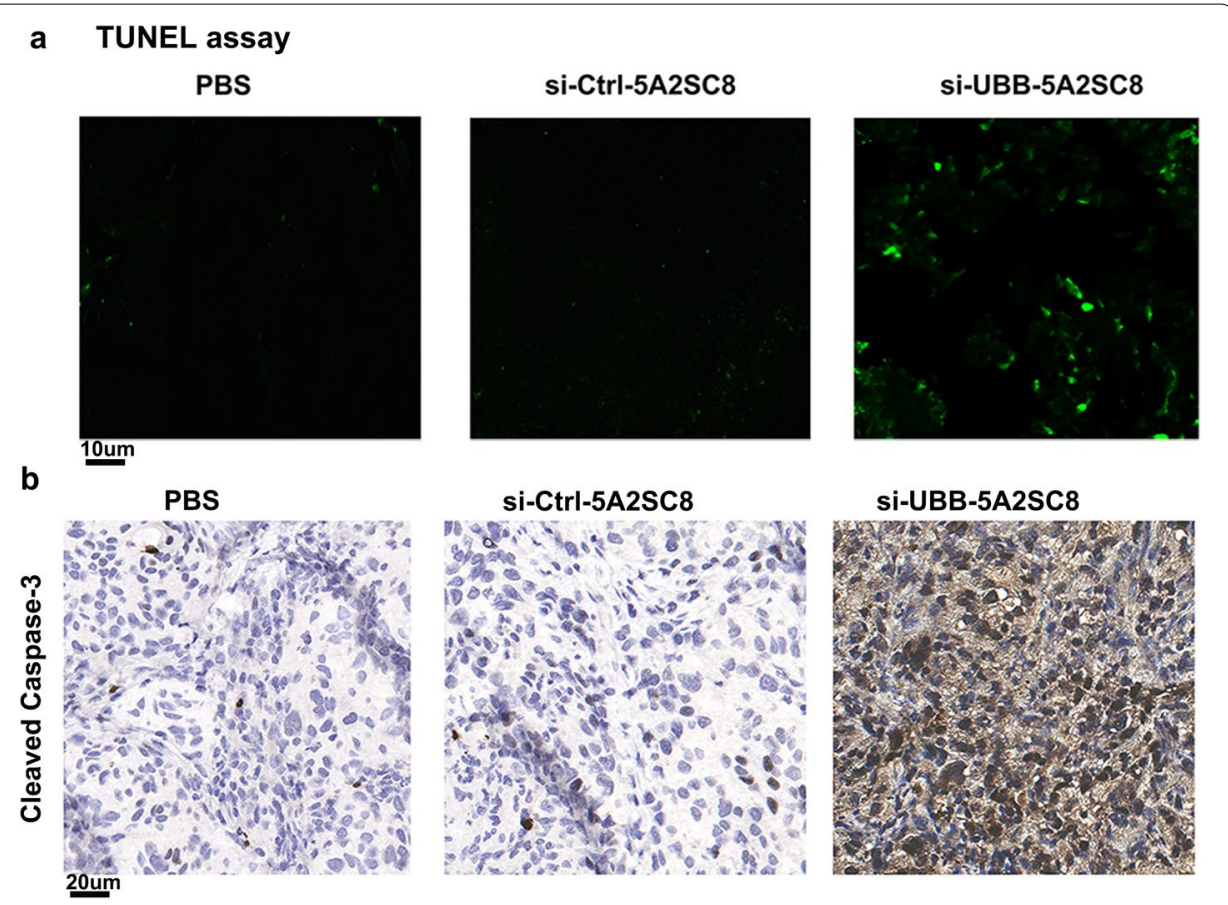

Fig. 7 Downregulation of UBB expression induced apoptosis in tumor-beard mice. TUNEL assay (a) and cleaved caspase-3 expression (b) were adopted to analyze the rate of apoptosis. In si-UBB-5A2SC8 treated group, the apoptosis was significantly increased, which means downregulation of UBB expression inhibited the tumorigenesis by inducing apoptosis. a: Scale bar $10 \mu \mathrm{m}$, b: scale bar $20 \mu \mathrm{m}$

non-toxic, which was demonstrated by $90 \%$ of cell viability. With the suitable zeta potential and ideal size, the nanoparticle can be efficiently uptaken by SKOV3-GFP cell and precisely deliver siRNA to the target location.

Polyubiquitin B is a member of the ubiquitin-proteasome pathway (UPP), which exerts a pivotal effect on homeostasis maintenance by regulation of proteolysis and misfolded proteins (Portilho et al., 2019). Cancer cells can produce proteins, which promote cell proliferation and inhibit cell death. UPP inhibition has been exploited as an anticancer strategy to balance protein synthesis and degradation (Kedves et al., 2017). It was reported that ubiquitin-conjugating enzyme E2B involved in nasopharyngeal carcinoma sensitivity to chemotherapy by mediating the ubiquitination of DNA methyltransferase (Hsu et al., 2018), and ubiquitin specific peptidase 1 regulates cellular autophagy by targeting unc-51 like autophagy activating kinase 1 (Raimondi et al., 2019). Meanwhile, deubiquitinase inhibitors were reported to have the potential to treat breast cancer, which was resistant to hormonal therapy (Xia et al., 2018). So we inferred downregulation of ubiquitin B may exert anti-tumor effect in ovarian cancer.

When we transfected SKOV3-GFP cell with si-UBB-5A2SC8, endogenous ubiquitin-B expression both in mRNA and protein level were inhibited, which was accompanied with arrest of cell migration and proliferation in vitro. In vivo distribution analysis demonstrated 5A2SC8 could efficiently deliver siRNA to tumor site and exhibited good anti-tumor activity via inducing apoptosis. Unfortunately, we did not add another group of free si-UBB to further verify the delivery effect of 5A2SC8 
nanoparticle in vivo study. Collectively, our study suggested that 5A2SC8 delivery of si-UBB could be a potential therapeutic strategy for ovarian cancer.

Our findings provide a solid theoretical foundation for 5A2SC8 delivery of siRNA to treat tumor therapy. Meanwhile, we demonstrated that ubiquitin-B was an underlying therapeutic target for anti-tumor therapy. Herein, we conclude 5A2SC8-based nanoparticle targeting ubiquitin-B is an innovative and promising method to conquer ovarian cancer. In our future study, we will use more models to further explore the high delivery efficiency of 5A2SC8 nanoparticles.

\section{Acknowledgements}

We appreciate Dr. Daniel Siegwart for providing us the 5A2SC8 particles, SKOV3-GFP cells and technical supports.

\section{Authors' contributions}

RL and SS developed the project. MX and LY performed experiments and wrote the manuscript. XL and SS supervised the work and edited the manuscript. All authors agreed with the final version of manuscript. All authors read and approved the final manuscript.

\section{Funding}

The present study was funded by National Natural Science Foundation of China (grant no. 81672496) and the National College Students Innovation and Entrepreneurship Training Program (grant no. CX18024).

\section{Availability of data and materials}

The authors declare that all of the data and material are freely accessible on reasonable request.

\section{Declarations}

Ethics approval and consent to participate

Not available.

\section{Consent for publication}

The paper was not under consideration for publication elsewhere, that its publication was approved by all authors.

\section{Competing interests}

The authors declare that they have no competing interests.

\section{Author details}

'Department of Gynecology and Obstetrics, The First Affiliated Hospital of Jinan University, 613 Huangpu Road West, 510630 Guangzhou, People's Republic of China. ${ }^{2}$ Department of Hematology, Guangzhou Institute of Pediatrics, Guangzhou Women and Children's Medical Center, Guangzhou, China. ${ }^{3}$ Department of Medical Ultrasound, Institute of Diagnostic and Interventional Ultrasound, The First Affiliated Hospital of Sun Yat-Sen University, Guangzhou, China

${ }^{4}$ Department of Clinical Medicine, Medical College of Jinan University, Guangzhou, China.

Received: 25 September 2021 Accepted: 19 January 2022

Published online: 29 January 2022

\section{References}

Aagaard L, Rossi JJ (2007) RNAi therapeutics: principles, prospects and challenges. Adv Drug Deliv Rev 59:75-86 Alvero AB, Kim D, Lima E, Sumi NJ, Lee JS, Cardenas C, Pitruzzello M, Silasi DA, Buza N, Fahmy T, Mor G (2017) Novel approach for the detection of intraperitoneal micrometastasis using an ovarian cancer mouse model. Sci Rep 7:40989

Barata P, Sood AK, Hong DS (2016) RNA-targeted therapeutics in cancer clinical trials: current status and future directions. Cancer Treat Rev 50:35-47

Bray F, Ferlay J, Soerjomataram I, Siegel RL, Torre LA, Jemal A (2018) Global cancer statistics 2018: GLOBOCAN estimates of incidence and mortality worldwide for 36 cancers in 185 countries. CA Cancer J Clin 68:394-424

Castillo PM, Jimenez-Ruiz A, Carnerero JM, Prado-Gotor R (2018) Exploring factors for the design of nanoparticles as drug delivery vectors. ChemPhysChem 19:2810-2828

Cheng Q, Wei T, Jia Y, Farbiak L, Zhou K, Zhang S, Wei Y, Zhu H, Siegwart DJ (2018) Dendrimer-based lipid nanoparticles deliver therapeutic FAH mRNA to normalize liver function and extend survival in a mouse model of hepatorenal tyrosinemia type I. Adv Mater 30:e1805308

Dragoni L, Ferrari R, Lupi M, Cesana A, Falcetta F, Ubezio P, D'Incalci M, Morbidelli M, Moscatelli D (2016) Small interfering RNA delivery through positively charged polymer nanoparticles. Nanotechnology 27:125102

Hsu SH, Chen SH, Kuo CC, Chang JY (2018) Ubiquitin-conjugating enzyme E2 B regulates the ubiquitination of O(6)methylguanine-DNA methyltransferase and $\mathrm{BCNU}$ sensitivity in human nasopharyngeal carcinoma cells. Biochem Pharmacol 158:327-338 
Kedves AT, Gleim S, Liang X, Bonal DM, Sigoillot F, Harbinski F, Sanghavi S, Benander C, George E, Gokhale PC, Nguyen QD, Kirschmeier PT, Distel RJ, Jenkins J, Goldberg MS, Forrester WC (2017) Recurrent ubiquitin B silencing in gynecological cancers establishes dependence on ubiquitin C. J Clin Invest 127:4554-4568

Kim DH, Rossi JJ (2007) Strategies for silencing human disease using RNA interference. Nat Rev Genet 8:173-184

Lees JC, Ellison J, Batchelor-McAuley C, Tschulik K, Damm C, Omanovic D, Compton RG (2013) Nanoparticle impacts show high-ionic-strength citrate avoids aggregation of silver nanoparticles. ChemPhysChem 14:3895-3897

Oh C, Park S, Lee EK, Yoo YJ (2013) Downregulation of ubiquitin level via knockdown of polyubiquitin gene Ubb as potential cancer therapeutic intervention. Sci Rep 3:2623

Ozcan G, Ozpolat B, Coleman RL, Sood AK, Lopez-Berestein G (2015) Preclinical and clinical development of siRNA-based therapeutics. Adv Drug Deliv Rev 87:108-119

Pinho SL, Pereira GA, Voisin P, Kassem J, Bouchaud V, Etienne L, Peters JA, Carlos L, Mornet S, Geraldes CF, Rocha J, Delville $\mathrm{MH}$ (2010) Fine tuning of the relaxometry of gamma-Fe2O3@SiO2 nanoparticles by tweaking the silica coating thickness. ACS Nano 4:5339-5349

Portilho LG, Duarte BCD, Queiroz FR, Ribeiro THC, Jeremias WJ, Baba EH, Coelho PMZ, Morais ER, Cabral FJ, Caldeira RL, Gomes MS (2019) Genome-wide identification, characterisation and expression profiling of the ubiquitin-proteasome genes in Biomphalaria glabrata. Mem Inst Oswaldo Cruz 114:e190052

Raimondi M, Cesselli D, Di Loreto C, La Marra F, Schneider C, Demarchi F (2019) USP1 (ubiquitin specific peptidase 1) targets ULK1 and regulates its cellular compartmentalization and autophagy. Autophagy 15:613-630

Xia X, Liao Y, Guo Z, Li Y, Jiang L, Zhang F, Huang C, Liu Y, Wang X, Liu N, Liu J, Huang H (2018) Targeting proteasomeassociated deubiquitinases as a novel strategy for the treatment of estrogen receptor-positive breast cancer. Oncogenesis 7:75

Zhou K, Nguyen LH, Miller JB, Yan Y, Kos P, Xiong H, Li L, Hao J, Minnig JT, Zhu H, Siegwart DJ (2016) Modular degradable dendrimers enable small RNAs to extend survival in an aggressive liver cancer model. Proc Natl Acad Sci U S A 113:520-525

Zhou Q, Yao X, Wu C, Chen S, Fan D (2020) Knockdown of ubiquitin-specific protease 53 enhances the radiosensitivity of human cervical squamous cell carcinoma by regulating DNA damage-binding protein 2. Technol Cancer Res Treat 19:1533033820929792

\section{Publisher's Note}

Springer Nature remains neutral with regard to jurisdictional claims in published maps and institutional affiliations.

- fast, convenient online submission

- thorough peer review by experienced researchers in your field

- rapid publication on acceptance

- support for research data, including large and complex data types

- gold Open Access which fosters wider collaboration and increased citations

- maximum visibility for your research: over 100M website views per year

At BMC, research is always in progress.

Learn more biomedcentral.com/submissions 